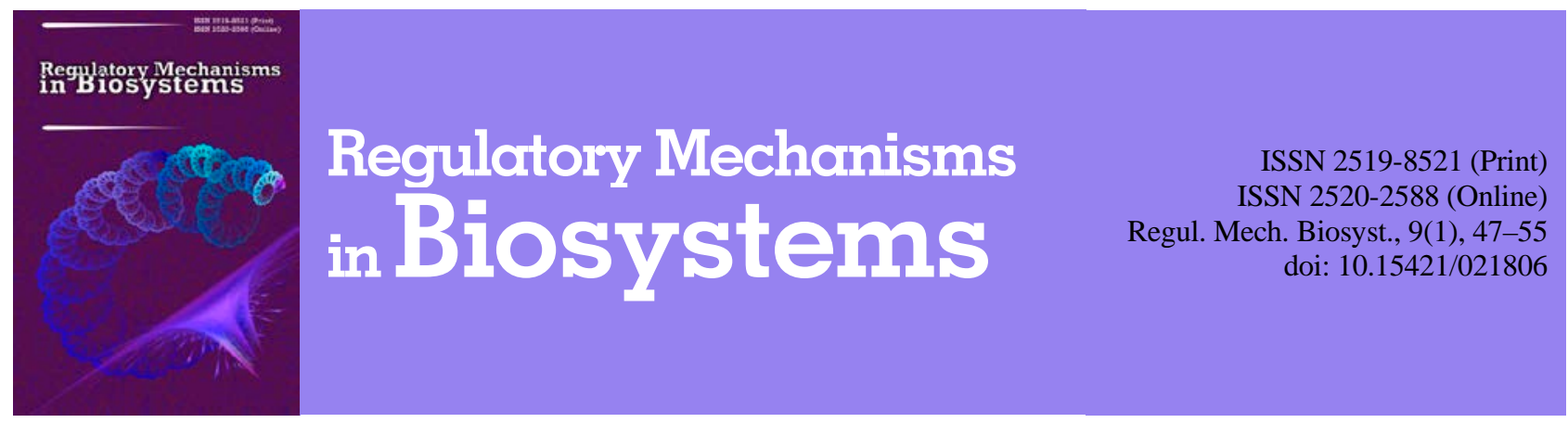

\title{
Modern magnetic immunoassay: Biophysical and biochemical aspects
}

\author{
O. Y. Galkin, O. B. Besarab, M. O. Pysmenna, Y. V. Gorshunov, O. M. Dugan \\ National Technical University of Ukraine "Igor Sikorsky Kyiv Polytechnic Institute”, Kyiv, Ukraine
}

Article info

Received 10.10.2017

Received in revised form 15.11.2017

Accepted 19.11.2017

National Technical

University of Ukraine

"Igor Sikorsky Kyiv

Polytechnic Institute",

Peremogy av., 37,

Kyiv, 03056, Ukraine.

Tel.: +38-044-240-90-36.

E-mail: a.galkin@kpi.ua

Galkin, O. Y., Besarab, O. B., Pysmenna, M. O., Gorshunov, Y. V., \& Dugan, O. M. (2018). Modern magnetic immunoassay: Biophysical and biochemical aspects. Regulatory Mechanisms in Biosystems, 9(1), 47-55. doi: 10.15421/021806

In this review article an analysis of the biochemical and biophysical aspects of modern magnetic immunoassay (MIA) is conducted and additionally the problems and perspectives of its application in biology, biotechnology and medicine are defined. Magnetic immunoassay should be considered as an evolutionary extension of the classical immunoassay. MIA can have many variants of modifications, similar to the classic immunoenzymatic assay. The key distinctive element of the MIA is the use of magnetic particles (MPs), which are usually nanoparticles. MPs in the MIA can act as a marker for detection, or the solid phase at which the immunochemical reaction takes place. MIA possesses basic advantages over classical immunoassay methods: thanks to the unique magnetic properties of the MPs and the ability to manipulate it in the external magnetic field, it is possible to increase the informative value of the analysis (first of all, sensitivity and specificity), as well as the rigid requirements for "purity" of tested samples. For the purposes of immunoassay, magnetic particles of size from 10 to $200 \mathrm{~nm}$ are important, since such particles are in a superparamagnetic state, in the absence of strong magnetic fields; they are not agglomerated in a liquid medium. The size of the spherical particle determines the rate of sedimentation and mobility in the solution. The outer polymeric membrane serves as a matrix in which the surface functional groups are added, and also protects the core of the metal from the external environment. The outer shell may also consist of agarose, cellulose, porous glass, silicon dioxide etc. There are several strategies for the synthesis of nanoparticles: mechanical (dispersion), physical (gas phase deposition), wet chemical methods (chemical comprecipitation, thermal decomposition, methods of micro emulsion, hydrothermal reactions) and physico-chemical methods. Also used are magnesite nanoparticles of biogenic origin. Magnetic particles may function, and this is important for immunoassay. Surface functional groups include carboxylic, amino, epoxy, hydroxyl, tosyl, and N-hydroxysuccinate-activated groups. Magnetic spherical particles usually interact with surface molecules such as streptovidine, biotin, protein A, protein G, and immunoglobulin etc. Directions and prospects of the development of methods of magnetic immunoassay are determined, mainly, by the development of methods for detecting or influencing magnetic particles. In this case, the classical methods of detection are electrochemical methods, electrochemiluminescence, fluorescence. More modern ones include giant magnetoresistance, superconducting quantum interference devices, surface-enhanced Raman spectroscopy, biosensors based on nonlinear magnetization, magneto-PCR immunoassay. The current trend is to combine or integrate the application of various biochemical, physical, molecular and genetic, physico-chemical detection methods. In fact, all of these benefits undoubtedly open up broad prospects for the practical application of MIA in biology, biotechnology and medicine.

Keywords: serologic diagnostics; magnetic particles; biochemical and physical methods

\section{Introduction}

Among the whole complex of methods of clinical laboratory diagnostics, methods of serologic diagnostics were among the first to be proposed and implemented in practical medicine. Serologic diagnostics remains extremely relevant to the present day. Serologic methods are used for diagnostics of infectious (bacterial, viral, fungal, parasitic), and non-infectious (oncological, endocrine, allergic) diseases. A significant proportion of diagnostic examinations carried out by the laboratory service relates precisely to serological tests. Serologic methods remain an indispensable part of the provision of sanitary and epidemiological well-being of the population (Galkin, 2014a).

One of the modern trends in laboratory medicine is the intensive use of various nanotechnologies, which should include methods using magnetic nanoparticles (MNPs). MNPs have already become an important tool in clinical laboratory diagnosis and medical imaging in vivo. Important prerequisites for the successful use of MNPs for medical purposes are their high "bioavailability", which is achieved both by the size of the particles, and the possibility of their functionalization (using covalent and non-covalent methods) and purposeful targeting under the influence of external magnetic fields, as well as the stability of physical characteristics (magnetization, size) and the possibility of their modeling depending on specific medical and biological tasks. The size of the magnetic particles (MPs) and the intensity of the external magnetic fields can be selected so that the effect of such MPs in living objects will be "physiological" (force can vary from $10^{-12}$ to $10^{-9} \mathrm{~N}$ ) (Aseri et al., 2015). Magnetic materials used in this case (compounds of iron, cobalt, nickel, etc.) are "technological", yet not all of them are characterized by an acceptable level of biocompatibility (when it comes to their in vivo use) (Wu et al., 2015; Foglia et al., 2017). All of the above, combined with the internal permeability of magnetic fields in human and animal tissues, offers an extremely wide range of possibilities for using MPs in biomedicine (Ghodbane et al., 2013; Issa et al., 2013). The use of magnetic nanotechnologies in laboratory diagnostics allows the elimination of the imperfections and limitations that are typical of traditional immunoassays (enzyme-linked immunosorbent assay, immunofluorescence, etc.) (Mani et al., 2011; Day et al., 2015; Wang et al., 2017a; Liao et al., 2017). In particular, it becomes possible to disclaim strict requirements to the "purity" of the tested material, but also expand the range of 
materials that can be tested; it is possible to increase the analytical sensitivity of the analysis, to improve other bioanalytical characteristics, etc. (Tsai et al., 2007; Lin et al., 2013; Li et al., 2014; Manera et al., 2017; Nie et al., 2017).

The purpose of our work is to analyze the current state of the use of magnetic immunoassay (MIA) in fundamental and applied research in biology, biotechnology and medicine.

\section{Problems and benefits of using MPs in immunoassay}

Widespread use of magnetic particles in immunoassay is due to the following circumstances. With the help of MPs, it is possible to increase the sensitivity and reduce the time of analysis by manipulating the particles with an external magnetic field, magnetic laundering and magnetic separation. In addition, as detected labels, MPs have advantages over traditional fluorescent and enzyme markers, whose application in opaque or highly dispersed biological media has a number of severe limitations. There are two main directions of use of MPs in immunoassay: firstly, MPs can act as a solid phase for immune complex formation, and, secondly, MPs can act as labels, for providing detection in the analysis (Fig. 1).

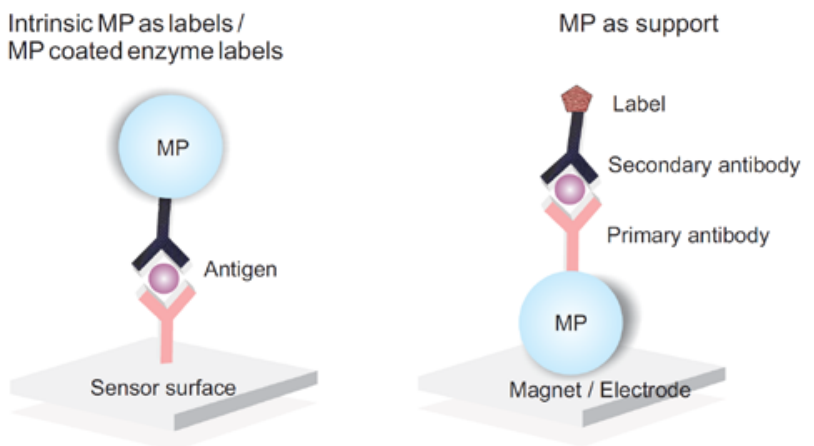

Fig. 1. Principal approaches to the use of MP in immunoassay (by Mani et al., 2011)

It should be noted that the studied bioanalysts (for example, biological fluids or food samples) are often complex colloidal systems, which, in turn, often leads to false-positive or false-negative results of immunoassay due to nonspecific reactivity of antibodies. Also it should be noted that in optical detection the color or autofluorescence of the sample can contribute to the recorded signal, increasing the noise and thus reducing the signal to noise ratio.

The above circumstances determine the widespread use of MPs for preliminary purification of the analyte (magnetic separation) (Choi et al., 2001; Nagasaki et al., 2007). With this approach, at the first stage, MPs with immobilized antibodies are added to the samples, and the antibodies recognize the wanted antigen. An external magnetic field is then applied and the antigen-enriched MPs can be used as a purified solid phase for further analysis (Fig. 1). Such a technique reduces the probability of non-specific binding and reduces the time of analysis (Gehring et al., 2004; Orlov, 2014). The use of magnetic particles as a solid phase increases the antigen's stability during immunoassay because, firstly, the number of washing steps is reduced and, secondly, the laundering process itself becomes more efficient due to the possibility of using an external magnetic field for solid phase manipulation (Choi et al., 2001; Orlov, 2014).

Fluorescent and enzyme labels used in immunoassay have a number of methodological limitations, which sometimes require laborious detection methods, which do not always have high reliability and reproducibility; therefore, it is very promising to use MPs in immunoassay as labels for detection (Orlov, 2014). Magnetic labels, as a rule, consist of nanoparticles of iron oxide in the size of 5-50 nm, which are enclosed in a polymeric membrane and form a particle in the size from $20 \mathrm{~nm}$ to $5 \mu \mathrm{m}$. Magnetic nanoparticles of such sizes have a unique magnetic property - lack of residual magnetization (so-called superparamagnetic nanoparticles). The phenomenon of superparamag- nity is widely used for biomedical purposes, e.g., in magnetic resonance tomography (Smith-Bindman et al., 2012), but the idea of using it for the detection of magnetic nanoparticles in immunoassay is relatively new (Luo et al., 2017; Xue et al., 2017; Sood et al., 2017). Magnetic particles have a number of advantages over standard optical labels. Firstly, since the level of the magnetic background in biological samples is usually negligible, using MPs can produce a very high signal to noise ratio. Secondly, the opacity or color of the samples does not affect the magnetic properties of MPs. Thirdly, the magnetic tags are stable, and their magnetic properties do not change. Fourth, with the help of an external magnetic field, it is possible to manipulate of MPs, to increase efficiency and reduce analysis time by magnetic stirring, washing and separating (Morozov et al., 2007; Nikitin et al., 2008a; Dittmer et al., 2010; Orlov, 2014). Currently, the use of magnetic nanoparticles as labels for immunoassay in combination with the active influence on it is popular.

Note that using MPs as a label has its own peculiarities and disadvantages. The most significant of them is the difficulty in achieving satisfactory analytical (validation) characteristics of the method, which primarily concerns linearity. Linearity is known to represent the ability of the technique (within the range of application) to give a value directly proportional to the concentration (amount) of the analyte in the sample (Galkin et al., 2015; Lutsenko et al., 2017). Such a situation, for example, occurs when determining staphylococcal enterotoxins in complex biological fluids. The use of MPs in combination with the fluidic force discrimination method for detecting enterotoxins using a flat chip and optical reading allowed high sensitivity to be achieved: the detection limit was $1 \mathrm{pg} / \mathrm{ml}$ and $1 \mathrm{fg} / \mathrm{ml}$ for multistage and semi-homogeneous analysis formats, respectively. However, with an increase in the concentration of antigen by 10 orders of magnitude, there was only a fourfold increase in the recorded signal (the signal increased by only $15 \%$ with a tenfold increase in the concentration of enterotoxins). The authors note that it is difficult to distinguish concentrations that differ by less than 10 times (Mulvaney et al., 2007; Mulvaney et al., 2009). Magnetic particle manipulation in combination with electrophoretic concentration of a toxin on the surface during active analysis leads to an increase in the optical signal by 1.7 times with a tenfold increase in the antigen concentration. Taking into account the distribution of observed signals, the authors conclude that the analysis can only provide a quailtative result, determining the presence or absence of a toxin. In addition, active analysis requires pre-centrifugation and desalting complex mediums (Shlyapnikov et al., 2012).

In this case it should be noted that all immunochemical methods are not always characterized by a satisfactory linearity, even providing mathematical transformation of the results of the study. Under these circumstances it is possible to move from the linearity as such to finding the proper concentration of analysis function in the samples under study (Chen et al., 2016; Giannetto, et al., 2017). One of the ways to overcome such a disadvantage is the following. When detecting enterotoxins, the number of MPs-labels can be determined not only optically, but also using biosensors based on the giant magnetic resistance (GMR). The successes in the development of GMR-biochips made them three orders of magnitude more sensitive than the enzyme-linked immunosorbent assay (ELISA). However, in order to double the signal, it is usually necessary to increase the concentration of antigen in order, and the full range of signal changes is one and a half order. Some approaches, such as enhancement of the GMR-signal by moving the magnetic particles into a zone with the highest sensitivity or optical reading of the magnetically activated particles, can increase the range of detected signals to two orders of magnitude (Kurlyandskaya et al., 2017; Rizzi et al., 2017; Wang et al., 2017a; Salek-Maghsoudi et al., 2018).

\section{Characteristics of magnetic particles and methods for obtaining them}

For the purposes of immunoassay, magnetic particles of size from 10 to $200 \mathrm{~nm}$ are important, since such particles are in a superparamagnetic state, in the absence of strong magnetic fields; they are not agglomerated in a liquid medium. Properties of magnetic nanoparticles and polymer clusters containing nanoparticles largely depend on the pro- 
perties of the magnetic material. One of the main properties is magnetic susceptibility $\chi_{\mathrm{m}}$, which characterizes the dependence of the magnetization of a substance on the intensity of the external magnetic field $\mathrm{H}$ (Tygai et al., 2014; Wu et al., 2016; Tang et al., 2017):

$$
\mathrm{M}=\chi_{\mathrm{m}} \times \mathrm{H} \text {. }
$$

The characteristic magnetization curve for MPs is shown in Fig. 2. At the same time, it is important that such value of magnetic field intensity HS corresponds to magnetic saturation MS. For immunoassay purposes, it is advisable to use MPs markers with greater magnetic susceptibility and higher magnetization of saturation with the same initial magnetic susceptibility of the particle (Orlov, 2014). This kind of characterization of the particles of iron oxides, and in particular of magnetite, is determined mainly by their crystalline structures and essentially depends on the conditions for the reaction to produce them (Rajput et al., 2016; Vidojkovic et al., 2017). The best magnetic properties of magnetite are achieved with an equal molar ratio of iron oxides (II) and (III).

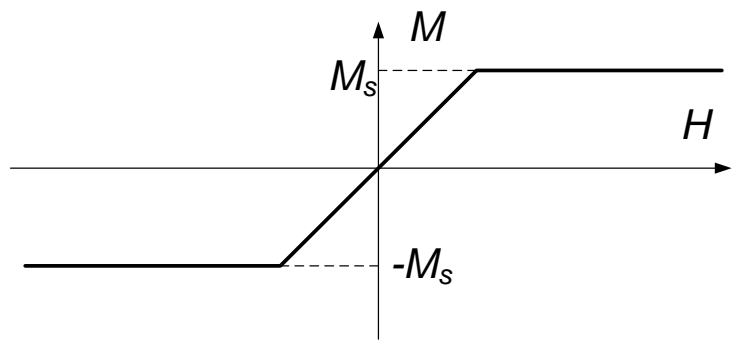

Fig. 2. Simplified MPs magnetization characteristic

It should be noted that at present, magnetic spherical particles of various types are available as commercial products for use in scientific and applied applications. Paramagnetic particles are most applicable to systems whose purpose is magnetic separation and transportation, since they acquire magnetism in the presence of a magnetic field but have zero magnetization in the absence of a magnetic field.

The most common examples of paramagnetic particles are magnesium oxide nuclei and non-magnetic polymer shells. It is the polymeric surfaces of such particles that provide the functional ability to chemically attach biomolecules. Metal oxides are used to create magnetic core more often than pure metals ( $\mathrm{Fe}, \mathrm{Co}, \mathrm{Ni}$ ), since they have higher oxidation resistance (Table 1 ). Polymer shells also stabilize magnetic particles, giving them elasticity and ability to swell.

The magnetic core can also consist of a set of paramagnetic nanoparticles located in the core of the polymer. Typically, nanoparticles of a size range from $100 \mathrm{~nm}$ to $50 \mu \mathrm{m}$ in diameter are commercially available. The size of the spherical particle determines the rate of sedimentation and mobility in the solution. The outer polymeric membrane serves as a matrix in which the surface functional groups are added, and also protects the core of the metal from the external environment. The outer shell may also consist of agarose, cellulose, porous glass or silicon dioxide (Day et al., 2015).

There are several strategies for the synthesis of nanoparticles: mechanical (dispersion), physical (gas phase deposition), wet chemical methods (chemical comprecipitation, thermal decomposition, methods of micro emulsion, hydrothermal reactions) and physico-chemical methods. Also used are magnesite nanoparticles of biogenic origin, forming certain types of bacteria.

\section{Table 1}

Saturation magnetization and magnetization susceptibility of some magnetic metal oxides (Philippova et al., 2011)

\begin{tabular}{lcc}
\hline \multicolumn{1}{c}{ Oxides } & Magnetization saturation, emu/g & Magnetization susceptibility \\
\hline$\gamma-\mathrm{Fe}_{2} \mathrm{O}_{3}$ & 74 & $-5 \times 10^{-6}$ \\
$\mathrm{Fe}_{3} \mathrm{O}_{4}$ & 84 & $+18 \times 10^{-6}$ \\
$\mathrm{Fe}_{2} \mathrm{O}_{3}-\mathrm{Fe}_{3} \mathrm{O}_{4}$ & $\sim 80$ & $+7 \times 10^{-6}$ \\
$\mathrm{CoO} \cdot \mathrm{Fe}_{2} \mathrm{O}_{3}$ & 65 & $-110 \times 10^{-6}$ \\
\hline
\end{tabular}

Wet chemical methods have been studied more widely than physical ones, since they can provide a higher level of control over the size, composition, magnetic properties, and the form of magnetic nanopar- ticles. This is especially important for screening cells in a liquid medium (Zhu et al., 2016).

The water-in-oil microemulsion (or reverse micelle) method provides the following: nanoparticles are formed by an isotropic dispersion or by mixing two liquids that form microdermas. Such micro domains are stabilized by the interphase film of the surface-active substance. This method can be considered as a derivative of deposition or a method of recovery with the difference that the reaction occurs in small droplets of water stabilized in an organic solvent. When mixing two microemulsions containing the necessary reagents, the micro domains come in contact and collapse again when stirred, resulting in the formation of a precipitate containing nanoparticles (Zhang et al., 2017; Beshkar et al., 2017). The low yield of nanoparticles, compared with other methods, as well as the need for a large amount of solvent, jeopardizes the efficiency and production scale.

Hydrothermal synthesis allows a wide range of nanostructured forms and compositions to be obtained. This synthesis method is based on phase change and separation, which occurs at the interface between liquid, solid and soluble phases (Wu et al., 2016). An example of the use of hydrothermal synthesis for the manufacture of monodispersed magnetic nanoparticles in the range from $200-800 \mathrm{~nm}$ is described. A mixture of iron salts (e.g., $\mathrm{FeCl}_{3}$ ), a high boiling point boiling substance (e.g. ethylene glycol), an electrostatic stabilizer (for example, sodium acetate) and a surfactant (for example, polyethylene glycol) are heated to $200^{\circ} \mathrm{C}$, and maintained at this temperature for 8-72 hours in a sealed autoclave made of stainless steel (Han et al., 2012).

Nanoparticles of metal oxides, in particular, magnetite $\mathrm{Fe}_{3} \mathrm{O}_{4}$ and magnetite $\gamma-\mathrm{Fe}_{2} \mathrm{O}_{3}$, are often synthesized with the use of alkaline coprecipitation of iron and iron salts (Mohapatra et al., 2010).

Synthesis of iron oxide nanoparticles with carboxymethyldextran and polyethylenimine polymeric membranes. Nanoparticles of iron oxide are synthesized by the method of co-precipitation (coprecipitation) of iron salts $\mathrm{FeCl}_{3}$ and $\mathrm{FeCl}_{2}$. The most common protocol for such synthesis involves the following. Use $5.9 \mathrm{~g} \mathrm{FeCl}_{3} \times 6 \mathrm{H}_{2} \mathrm{O}$ and $2.15 \mathrm{~g} \mathrm{FeCl}_{2} \times 4 \mathrm{H}_{2} \mathrm{O}$, mixed in $100 \mathrm{ml}$ of degassed water, followed by addition of $12.5 \mathrm{ml}$ of $30 \% \mathrm{NH}_{4} \mathrm{OH}$. The solution is heated to $85{ }^{\circ} \mathrm{C}$, and incubated for 2 hours. The formed suspension of particles is washed with $2 \mathrm{M} \mathrm{HNO}_{3}$ for peptizing the particles and also three times by degassed water. Then the aggregates are removed using a magnet, and the nanoparticles in the supernatant are covered with polymers. A solution of carboxymethyldextran (CMD) or polyethylenimine $25 \mathrm{kDa}$ at a concentration of $300 \mathrm{~g} / \mathrm{l}$ is added to nanoparticles to a final concentration of $50 \mathrm{~g} / \mathrm{l}$ and incubated for 4 hours at $80^{\circ} \mathrm{C}$. The resulting particles are washed off the free polymer by centrifugation at 16,800 $\mathrm{g}$ for 1-3 hours (Mohapatra et al., 2010).

Synthesis of ferritic nitride nanoparticles coated with a carboxymethyldextran polymer shell. Nanoparticles of ferritic acid are most often synthesized according to the method (Shevchenko et al., 2017), which provides the following. $8.85 \mathrm{~g} \mathrm{FeCl}_{3} \times 6 \mathrm{H}_{2} \mathrm{O}$ is used in $100 \mathrm{ml}$ of degassed water; the salt is precipitated with addition of $12.5 \mathrm{ml}$ of $30 \% \mathrm{NH}_{4} \mathrm{OH}$ and incubated for $2 \mathrm{~h}$ at $90{ }^{\circ} \mathrm{C}$. Subsequently peptizing the particles using $0.6 \mathrm{M} \mathrm{HNO}_{3}$ for 10 minutes is performed and washed with $\mathrm{HNO}_{3}$ by three-times centrifugation, after which the particles are coated by $\mathrm{CMD}$ at $80{ }^{\circ} \mathrm{C}$ for 4 hours, followed by threetimes centrifugation to wash off the unbound polymer.

Synthesis of golden nanoparticles. Golden nanoparticles are synthesized by the reduction of the hydrochloric acid of $\mathrm{HAuCl}_{4}$ with sodium citrate (Santhoshkumar et al., 2017).

Biosynthesis of magnetic particles. Intracellular biogenic magnetic nanoparticles (BMNPs) in the form of crystals of magnetite, magma and grazhite are found in many organisms, including bacteria, insects, mushrooms, fish, birds, animals and others (Gorobets et al., 2017). BMNPs are also found in normal tissues of the brain, liver, heart, spleen, and also in human tumor tissues (Gorobets et al., 2014a). The genetic regulation of the synthesis of these nanoparticles is thoroughly studied solely for magnetotaxis bacteria. The so-called genes of the magnetosomal islet of such bacteria responsible for the synthesis of magnetos are revealed. The use of natural magnetic properties of microorganisms containing BMNPs, as well as the development of new 
technologies for the creation of synthetic analogues of BMNPs in vitro, using biomineralization proteins, will allow the acquisition of magnetic nanoparticles with controlled parameters, which is an extremely important task for many technologies: for immunoassay, purposeful delivery of medicinal preparations, magnetic separation of biological media (Gorobets et al., 2013a, 2013b, 2014b).

Enzymatic synthesis of magnetic nanoparticles. The first in vitro enzymatic synthesis of paramagnetic and antiferromagnetic nanoparticles toward magnetic ELISA reporting has been reported (Kolhatkar et al., 2015). With our procedure, alkaline phosphatase catalyzes the dephosphorylation of l-ascorbic-2-phosphate, which then serves as a reducing agent for salts of iron, gadolinium, and holmium, forming magnetic precipitates. The nanoparticles were found to be paramagnetic at $300 \mathrm{~K}$ and antiferromagnetic under $25 \mathrm{~K}$. Although weakly magnetic at $300 \mathrm{~K}$, the room-temperature magnetization of the nanoparticles found here is considerably greater than that of analogous chemicallysynthesized samples. This approach of enzymatically synthesizing magnetic labels reduces the cost and avoids diffusional mass-transfer limitations associated with pre-synthesized magnetic reporter particles, while retaining the advantages of magnetic sensing.

\section{Methods of magnetic particles functionalization}

Surface functional groups include carboxylic, amino, epoxy, hydroxyl, tosyl, and N-hydroxy succinate-activated groups (Fig. 3). Magnetic spherical particles usually interact with surface molecules such as streptovidine, biotin, protein A, protein G, IgG, IgE, and IgM.

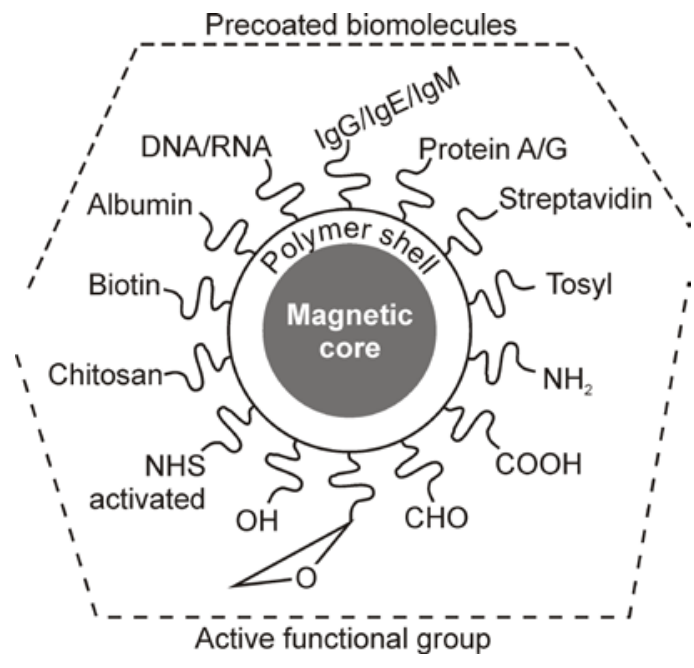

Fig. 3. Structure of MPs and surface functional groups

(by Mani et al., 2011)

Surface functional groups can be activated by EDC-coupling chemistry for carboxylates and glutaraldehyde for amines in order to further interact with functional groups of biomolecules (EDC = l-ethyl3-(3-dimethylaminopropyl) carbodiimide). Surface tosyl, N-hydroxy succinate-activated and epoxy groups can be used to attach biomolecules without the use of cross-linking agents. Particles pre-coated with streptavidin can interact with biotinyled biomolecules. Magnetic particles coated with protein A can selectively bind to Fc-regions of antibodies for the purpose of targeted immobilization (Mani et al., 2011).

Magnetic particles consisting of paramagnetic nanoparticles that are in the polymer matrix of the nucleus can have a multi-domain magnetic structure with residual magnetic moments. Such a structure of the nucleus of a magnetic nanoparticle can lead to magnetic clusterization due to the induced magnetism of neighboring particles. At room temperature, real paramagnetic particles, whose nuclei are constructed of iron oxide, should have radii in the low range $\left(\sim 10^{-9} \mathrm{~m}\right)$. In this way, usually, particles of $0.1-3.0 \mu \mathrm{m}$ in diameter, whose nuclei are constructed of nanoparticles of iron oxide, are coated with a polymer. Such particles are characterized by clusterization in the dispersion due to magnetic interactions between particles (Hermanson, 2008; Mani et al., 2011).
Covalent conjugation of nanoparticles with proteins. The nanoparticles coated with the polymers with HOOC-groups covalently bind to protein molecules using EDC as a crosslinking agent (crosslinker). During this reaction, an intermediate product, the derivative of O-acylizoic acid is formed, and it undergoes a nucleophilic attack on the amino group of the protein, resulting in the formation of a stable amide bond between the amino group and the carboxyl group on the surface of the particle. To increase the stability of the active intermediate and reduce the probability of hydrolysis EDC should be used together with N-hydroxysulfosuccinimide (Nhydroxysulfosuccinimide, sulfo-NHS). This reaction is carried out in two steps: first, the nanoparticles are activated with EDC/sulfo-NHS in the MES buffer (based on 2-(Nmorpholino)ethanesulfonic acid), then, after removing excess crosslinkkers centrifuged or using a magnetic tripod, the protein is added in the appropriate buffer. Optimal protein ratio: nanoparticles: EDC/sulfoNHS should be selected experimentally for each type of conjugate. In order to prevent the aggregation of the nanoparticles during the reaction, it is advisable to periodically process the ultrasound bath. The reaction is usually carried out for at least two hours, after which the excess of the unreacted protein is removed by centrifugation (for particles $<150 \mathrm{~nm}$ ) or by magnetic separation (for particles $>150 \mathrm{~nm}$ ) (Rusling et al., 2010; Shipunova et al., 2013).

\section{Methods for detecting of systems based on MPs}

Requirements for magnetic particles and their synthesis depend on the methods by which they are planned to be used in bioanalysis. Moreover, the control of the synthesis of magnetic particles and the selection of conditions of immunoassay should be carried out using the registration device. Below we provide characteristics of the main approaches for detecting magnetic particles in immunoassay - when MPs are a label (giant magnetoresistance, superconducting quantum interference devices, electrochemical methods et al.), and when MPs are a basis for the immune complex formation (electrochemical methods, electrochemiluminescence, fluorescence, magneto-PCR immunoassay et al.).

Giant magnetoresistance (GMR) is the quantum and mechanical effect observed in thin metal films, consisting of ferromagnetic and conductive non-magnetic layers alternating between them. The effect is a significant change in the electrical resistance of such a structure when the mutual direction of magnetization of the neighboring magnetic layers is changed. The direction of magnetization can be controlled, for example, by the influence of the external magnetic field (Fert, 2008). In the absence of an external field, the magnetic moments of the neighborring ferromagnetic layers are oriented antiparallelly, which ensures high electrical resistance of the structure. If the GMR sample is placed in an external magnetic field, the magnetic moments of all ferromagnetic layers are aligned along the directions of this field, which causes a decrease in the structure's resistance. At zero value of the external field the resistance is maximal. With increasing field, the resistance initially falls linearly, and then goes to saturation. Depending on the conditions and material, the maximum resistance of the sensor may be more than minimum value by $1.2-5.0$ times. Thus, by changing the resistance of the GMR-sensor one can judge the presence of an external magnetic field on it.

For the detection of magnetic particles, the GMR-structure is placed in an external magnetic field perpendicular to the surface of the sensor. Such an external field does not affect the structure's resistance, since the resistance change can only be caused by the field that lies in the sensor plane. The magnetic particle on the surface of the GMS structure causes a change in the distribution of the magnetic field so that the component of the external field appears along the sensor plane. Consequently, the resistance of the GMR-structure decreases when MPs are on its surface. Thus, using the GMR-structure, the presence of magnetic particles on the surface of the sensor can be detected. For immunoassay, the surface of the GMR sensor is modified to immobilize antibodies that will specifically bind to the antigen tested when the test samples are passed (Fig. 4). After that, a solution of MP, coated with antibodies to another antigen epitope, is applied to the sensor surface. By changing the resistance of the GMR structure, the amount of MPs that are connected 
to the sensor surface (Kim et al., 2013) is calculated. It should be noted that the bioanalytical application of GMR sensors has a number of limitations. A magnetic sensor detects distortion of the external magnetic field by a particle. However, the spatial scale of such distortion can be compared with the size of the particle. Therefore, with the standard technologies of GMR-sensors creating, only magnetic clusters of micron- size, comparable to the dimensions of the sensitive sensor element, are used. Such clusters are much larger compared to molecules that are recognized or revealed during the analysis, which imposes a number of restricttions on the use of such technology. In addition, the magnetization of small magnetic particles is small enough, so the signal is recorded with a low signal to noise ratio (Orlov, 2014; Rizzi et al., 2017; Crespo et al., 2018).

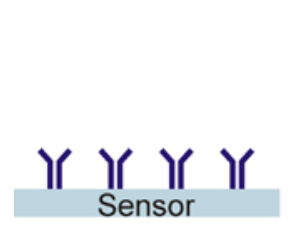

a

II Capture antibody

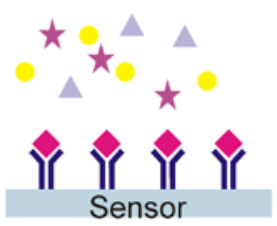

b

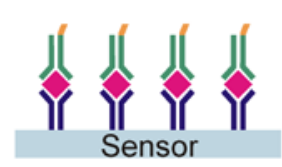

C

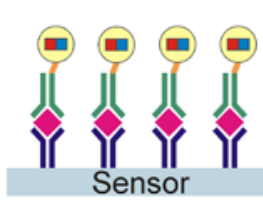

d

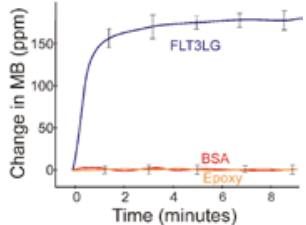

e

Fig. 4. A schematic of magneto-nanosensor biochip immunoassay: (a) Capture antibodies are immobilized covalently on the sensor surface. (b) Target antigens are captured and noncomplementary antigens are subsequently washed away. (c) Addition of biotinylated detection antibody forms a sandwich structure. $(d)$ Streptavidin-coated magnetic nanoparticles bound to the biotinylated detection antibody produce stray magnetic field.

(e) An example of real-time binding curve showing the change in magnetoresistance (MR) in parts per million (ppm) over time for 500 pg/ml Flt3lg (blue) compared with BSA negative control (orange) and epoxy reference (red) (by Kim et al., 2013)

Superconducting quantum interference devices (SQUIDs). SQUIDs are ultrasensitive magnetometers used to measure very weak magnetic fields. SQUIDs have a record high sensitivity that reaches $5 \cdot 10^{-33} \mathrm{~J} / \mathrm{Hz}$ (sensitivity to a magnetic field of about $10^{-13} \mathrm{~T}$ ). For longterm measurements of averaged values within a few days, sensitivity values of $5 \cdot 10^{-18} \mathrm{~T}$ can be achieved (Drung et al., 2007). There are two types of SQUIDs: based on direct current and high-frequency devices. The work of SQUIDs on alternating current is based on the nonstationary Josephson effect and uses only one Josephson contact. Many of the experiments in fundamental physics and measurements in biomagnetism, including the measurement of excessive signals, are performed using alternating current SQUIDs. The exceptionally high sensitivity to the magnetic flux relies on the entire spectrum of medical applications of SQUIDs (magnetoencephalography, magnetogastrography, magnetic marker monitoring, and heart research). There are also considerations regarding the application of SQUIDs in a quantum computer as qubits (Vesanen et al., 2013).

Unlike traditional magnetometers in which SQUIDs are used as passive low-frequency or permanent magnetic field sensors, an alternating current of the microwave frequency circulating around the SQUID ring is used in a scanning SQUID microscope when a constant voltage occurs on its Josephson junctions (a non-stationary Josephson effect). The basic principle of the fact that the microwave current flows in the SQUIDs ring is easier when a corresponding sample is next to it (Vesanen et al., 2013).

Recently SQUIDs-magnetometers have been used for immunoassay (Nakatani et al., 2012; Saari et al., 2015; Park, 2016; Rong et al., 2016; Liao et al., 2017). Thus, the use of magnetic markers in combination with the detection of SQUIDs allowed reducing detection in a standard ELISA. Another example of the application of SQUID technology is the detection of bacteria by the time of magnetic relaxation. In this approach, the magnetic particles are added to the model under study, immobilized antibodies on the surface, bacteria specifically recognized for the recognition. Further, this template is placed in a homogeneous external magnetic field. The magnetic moments of the parts while guided parallel to the field. If you remove the source of the external magnetic field, the particles will return to the state of minimum energy, when the total magnetic moment of the system will be zero. In this case, two mechanisms of relaxation of the magnetic moment will compete: Brownian and Néel. Brunov's mechanism is associated with the physical turn of himself, and Néel's with the turning of the magnetic moment inside the stationary particle (Wang et al., 2015, 2016).

For free particles, a fast Brownian relaxation mechanism will dominate, but for particles that bind to bacteria, the slower Néel mechanism becomes predominant, since the rotation of such particles is complicated. Thus, the total time of magnetic relaxation will depend on the number of parts that are associated with the bacteria, and hence the concentration of the bacteria themselves in the sample. With SQUIDs, the dependence of the magnetic signal on time is measured and the relaxation time is calculated. At the time of magnetic relaxation, the presence and concentration of bacteria in the sample are judged. An essential advantage of using the method of magnetic relaxation is that the analysis is carried out in one step, without any blurring steps. Nevertheless, there are significant difficulties: the need for cryogenic cooling, labor-intensive calibration of the method, etc., which does not allow wide use of this approach in biochemical diagnostics (Eberbeck et al., 2008; Orlov, 2014).

Biosensors based on nonlinear magnetization. The method of detecting magnetic nanoparticles by their nonlinear magnetization is based on the effect on the particles of the external alternating magnetic field at two frequencies. For the construction of such biosensors, the main consideration is the choice of frequency and amplitude of the corresponding components of the external field. The amplitude of field intensity $\mathrm{H} 1$ for the fields with a lower frequency should be greater than the magnitude of field intensity of saturation HS of MPs core. In this way, the low-frequency component of the magnetic field will periodically block the ability of the magnetic particles to further magnetization. The equivalent field intensity $\mathrm{H}(\mathrm{t}$ ) (Fig. 5c) consists of both the sum of the low-frequency components H1 (t) (Fig. 5a) and the high-frequency component $\mathrm{H}_{2}(\mathrm{t})$ (Fig. 5b). Under conditions of the MPs magnetization for the simplified characteristic (Fig. 5), and no consider the magnetic hysteresis phenomenon, we obtain a graph of MPs magnetization (Fig. 5d), which is vertically limited by the values of saturation magnetization \pm MS. The resultant induction signal associated with the presence of MPs will be nonlinearly modulated by both frequencies (Manera et al., 2017; Nikitin et al., 2017). Subsequently, the mathematical analysis of the results recorded by the sensor, the decomposition of the signal in the Fourier series, the selection of the component of high-frequency oscillations, and the corresponding idealized (no-noise) $\mathrm{m}<2>$ (t) curve are shown in Fig. 5e.

In real conditions, the high-frequency component of the signal will have significant noise, caused, in particular, by the presence of constructive elements of the sensor, having magnetic properties. One of the approaches to noise isolation is the use of MPs with standardized magnetization characteristics, which will have an average value of the existence of a high frequency component of the detected signal for half the period of the low frequency signal. What makes it possible to isolate from the signal only the high-frequency component, which has the required duration (caused by MPs itself). This approach is a rather reliable method for registering minor changes, even in the presence of significant background noise - in fact, there is a possibility to significantly increase the sensitivity of the analysis. The promise of such a method is due to the fact that it can be used in vivo (Nikitin et al., 2008b). 
Surface-enhanced Raman spectroscopy (SERS) is a surface-sensitive technique that enhances Raman scattering by molecules adsorbed on rough metal surfaces or by nanostructures such as plasmonic-magnetic silica nanotubes. The enhancement factor can be as much as $10^{10}$ to $10^{11}$ which mean the technique may detect single molecules (Xu et al., 2013).

Surfaces with nanoparticles, prepared for the detection of Raman scattering, are used to detect biomolecules, and therefore can determine the presence of proteins and biological fluids. This technique was used to detect urea in plasma, and can be considered as a candidate for a new generation of cancer diagnostic methods. The ability to analyze the composition of the mixture at the nanoscale makes the surfaces prepared for SERS promising in environmental studies, pharmaceutics, in the analysis of real experiments, for the detection of narcotic substances and explosives, the analysis of food quality, the detection of individual algal cells, etc. SERS in combination with plasmonic elements can be used for highly sensitive methods for detecting the interaction between biomolecules (Yang et al., 2013; Han et al. 2014; Li et al., 2014; Pallaoro et al., 2015; Lin et al., 2016; Xu et al., 2016).

Electrochemical biosensors containing capture antibodies and secondary antibodies with a label (Fig. 1) are characterized by high indicators of informative analysis (Mani et al., 2011). Electrochemical sensors based on MPs are widely used in medical devices for in vitro diagnosis, and most of them are based on capture ELISA (Tang et al., 2007; Tsai et al., 2007).

a)

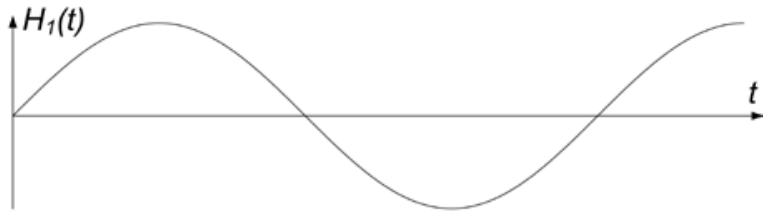

$\mathrm{H}_{2}(\mathrm{t})$

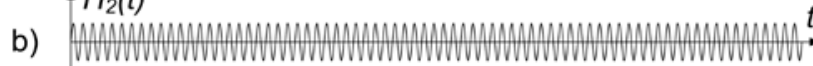
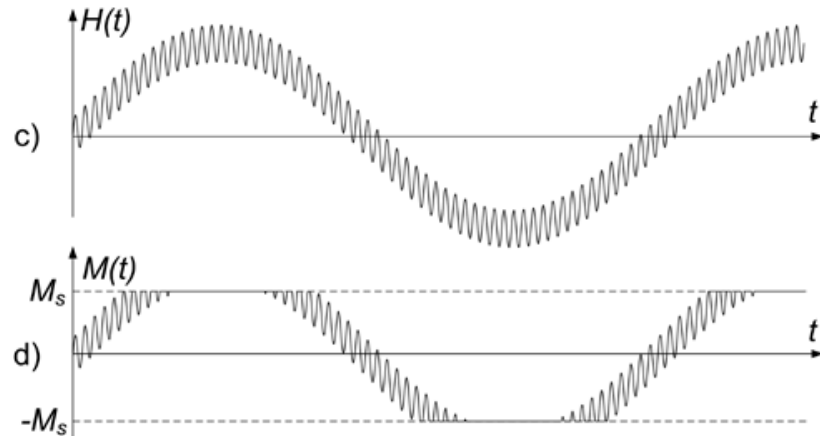

e)

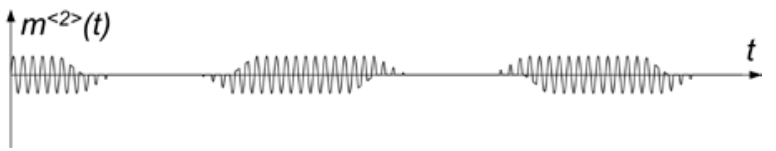

Fig. 5. The principle of detecting MPs on the basis of non-linear remagnetization

Electrochemiluminescence (ECL) detection envisages electrochemically generated light emission. As luminescent label most often are used tris (2,2'-bipyridyl) ruthenium (II). Often, such analytical systems contain avidin-biotin reagents based on the capture ELISA principle and have a quite satisfactory bioanalytical characteristic, which brings opportunities for their wide practical use (De Roeck et al., 2017; Nie et al., 2017; Wang et al., 2017b).

Magneto-PCR based immunoassay. This method can be considered as an analogue of ELISA in various modifications (including using the avidin-biotin signal amplification system) in which the label is not an enzyme but a DNA sequence. MPs function as a solid phase. Obviously, such an analysis is more cumbersome, compared with immuno-PCR, but it can provide even more impressive indicators of informativity, in particular, analytical sensitivity (Malou et al., 2011). For example, the authors managed to reach the limits of detecting the surface antigen of hepatitis B virus at $320 \mathrm{pg} / \mathrm{ml}$ (traditional ELISA test- kits have a sensitivity of about $10 \mathrm{ng} / \mathrm{ml}$ ) (Wacker et al., 2007). Consequently, such analytical systems are designed to determine the small concentrations of bioanalys, including in complex materials.

Magnetic immunoassay automation. An important precondition for the widespread use of MIA in clinical laboratory diagnostics is possibility to automate and standardize of the analysis. The latter is related with the standardization of hardware implementation of the method. The most original automated application of MIA addresses the technology of epitopon mapping. Epitope mapping enables one tos obtain genetic engineering peptides (proteins) and their subsequent study as potential antigenic determinants. At present, many variants of this methodological approach have been developed, in particular: phage and bacterial display, site-directed mutagenesis, mapping of recombinant proteins with tag (Galkin, 2014b). The methodological principles of use of MPs in epitope mapping technologies were formed at the end of the 20th century (Kala et al., 1997; McConnell et al., 1999). Librarybased display technologies have been staggeringly optimized since their appearance in order to mimic the process of natural molecular evolution. Display technologies are essential for the isolation of specific high-affinity binding molecules (proteins, polypeptides, nucleic acids and others) for diagnostic and therapeutic applications in cancer, infectious diseases, autoimmune, neurodegenerative, inflammatory pathologies etc. Applications extend to other fields such as antibody and enzyme engineering, cell-free protein synthesis and the discovery of protein-protein interactions. Phage display technology is the most established of these methods but more recent fully in vitro alternatives, such as ribosome display, mRNA display, cis-activity based display and covalent antibody display, as well as aptamer display and in vitro compartmentalization, offer advantages over phage in library size, speed and the display of unnatural amino acids and nucleotides. Altogether, they have produced several molecules currently approved or in diverse stages of clinical or preclinical testing and have provided researchers with tools to address some of the disadvantages of peptides and nucleotides such as their low affinity, low stability, high immunogenicity and difficulty to cross membranes (Galán et al., 2016).

When setting MIA usually requires the presence of a microtiter plate, a reader, a magnetic separator (used for washing the wells of the tablet), a thermostat, a multichannel pipette. An original example is the use of a pin-based magnetic particle processor to automate the method. The processor can accommodate several microtiter plates filled with different washing buffers and with different incubation periods. Consequently, the processor application ensures the standardization of such parameters as washing conditions, incubation time, and parallel tests in different buffers. Separate stages of magnetic separation occur when magnetic particles are transferred between the wells and rodshaped magnets, coated with a plastic coating through successive seizure and release movements. Operating mode of magnetic particle processors: the rod-shaped magnet is covered by a plastic cap and moves into a solution containing suspended magnetic beads; moving slowly up and down, the beads are attracted to the cover, and by moving the covered magnet to the next position, the beads are transferred to a new solution; once the magnet is removed from the cap, the beads are slowly suspended again; the magnet head and plastic covers are raised to the starting position to proceed to the next stage of the process (Konthur et al., 2010).

\section{Comparative characteristics of MIA and its prospects}

The use of magnetic particles provides a number of advantages, including the ease of separation and the suitability for automation. After coating the magnetic particles with the ligand, they become suitable for selective capture and distribution of various molecular particles. In this case, undesired components, large or fibrous particles, and a viscous matrix of the sample can be washed after a simple stage of magnetic particle distribution. Thus, the high efficiency of magnetic separation prevents the effect of non-target molecules that create the background and provides the most sensitive detection of target molecules.

Magnetic particles are often used to detect antibodies/antigens for several reasons. The use of MPs makes it possible to increase the 
surface area relative to the stable fluid volume in the well of a 96-well plate, thereby facilitating the interaction between antigen and antibody in a small volume (Lin et al., 2013). Increasing the area of the surface and uniform distribution of the particles throughout the sample provides the speed and sensitivity of determining the content of the molecules in question at low concentrations. In addition, magnetic spherical particles can be easily and quickly absorbed at the bottom of the well plate, and can be separated from the medium using a magnetic field (Lin et al., 2013). The method of magnetic ELISA is simple and fast, and, due to the very low non-specific binding background, it requires very small amounts of magnetic particles and a ligand. Preparation of magnetic parts is carried out easily and quickly. Magnetic ELISA also provides the availability of certain epitopes on the surface of the granules, that is, the availability of primary antibodies, in the case of setting sandwich variants of the method (Kourilov et al., 2002). The limitation in the formulation of a magnetic ELISA still is the interpretation of the results obtained at very low concentrations of target substances in the samples (Burgos-Ramos et al., 2012).

The main advantages of MIA compared with the traditional ELISA are as follows. First, the kinetics of the reaction of homogeneous small particles of magnesium added to the solution of the sample occurs quickly and effectively. The kinetics of the reaction when formulated with the usual ELISA is much slower, since only the bottom layer of the solution, captured on the surface of the polystyrene plate, directly contacts the test substance in the sample. Secondly, removing unbound reagents is more thorough when using homogeneous MPs, since the whole surface of the magnetic particles in the suspension is washed out. This approach provides the ability to remove most unbound reagents, which helps to achieve a lower nonspecific background, which in turn, improves the sensitivity of the analysis. Thirdly, even coverage ensures the delivery of particles with similar properties to all wells. During adsorption of molecules on the surface of the plate the phenomenon of sticking to the walls of the hole tablet may occur, which will create a background. Fourthly, the surface properties of the magnetic particles can be modified to maximize and/or orientate the molecules attached to their surface. Different molecules can be attached to the surface of the particles by passive adsorption or by covalent binding as needed. Fifthly, only magnetic particles can capture certain of the molecules analyzed in the suspension, ignoring other components in the solution. As a result, the target molecules can be concentrated in the precipitate for a few minutes without centrifugation. The sediment of magnetic particles with entrapped molecules analyzed can be transferred to the well of the plate for analysis.

Magnetic particles are characterized by numerous properties that make them suitable for widespread use in various fields - from visualization to drug delivery: easy to fabricate, manipulate in fluid, as well as a wide range of commercially available diameters of magnetic particles, ranging from nanoparticles $(50 \mathrm{~nm})$ to microparticles (up to ten microns) (Svobodova et al., 2015).

Magnetic particles have a wide range of applications: positive and negative selection of cells, allocation of molecular complexes. Due to the speed and ease of staging magnetic ELISA, this type of ELISA has found its application for clinical purposes (Hoyoung et al., 2013). Magnetic ELISA has been used for quantitative evaluation of immunoglobulins, rapid detection of circulating antigens, determination of cyclosporin A, and for other purposes. Selection of cells by means of magnetic particles has a certain advantage. The method is fast and easy to execute and does not require complicated hardware design. In addition, the method practically does not affect the viability of cells, it requires only very small amounts of the ligand, and can be performed in sterile conditions. Activated balls adsorb ligands, are economically affordable and can bind a large variety of molecules: antibodies, antigens, hormones, DNA and RNA. Thus, the MIA absorbed on the surface of the magnetic particle by a specific antibody is an excellent way to test the adsorption of the appropriate ligand and verify the availability of the appropriate epitopes. Another application of MIA is the detection of mutations in medical genetics. This method has been used for genotyping in samples from patients with predisposition to thrombophilia and detecting fusion transcripts of chromosomal translocations in children with acute lymphoblastic leukemia (BurgosRamos et al., 2012). MIA can also be used to detect pathogens and toxins present in food, water analysis (Orlov et al., 2013).

\section{Conclusions}

Magnetic immunoassay should be considered as an evolutionary extension of the classical immunoassay. MIA can have many variants of modifications, similar to the classic immunoenzymatic assay. The key distinctive element of the MIA is the use of magnetic particles, which are usually nanoparticles. MPs in the MIA can act as a marker for detection, or the solid phase at which the immunochemical reaction takes place.

MIA possesses two basic advantages over classical immunoassay methods: thanks to the unique magnetic properties of the MPs and the ability to manipulate it in the external magnetic field, it is possible to increase the informative value of the analysis (first of all, sensitivity and specificity), as well as the rigid requirements for "purity" of tested samples.

Directions and prospects of the development of methods of magnetic immunoassay are determined, mainly, by the development of methods for detecting or influencing magnetic particles. In this case, the classical methods of detection are electrochemical methods, electrochemiluminescence, fluorescence. More modern ones include giant magnetoresistance, superconducting quantum interference devices, magneto-PCR immunoassay. The current trend is to combine or integrate the application of various biochemical, physical, molecular and genetic, physico-chemical detection methods. Such complex approaches, on the one hand, allow one to achieve the best bioanalytical characteristics of the analysis, and, on the other hand, complicate the hardware design of the methods, which is not always favorable for practical use. In fact, all of these benefits undoubtedly open up broad prospects for the practical application of MIA in biology, biotechnology and medicine.

\section{References}

Aseri, A., Kumar, G. S., Nayak, A., Trivedi, S. K., \& Ahsan, J. (2015). Magnetic nanoparticles: Magnetic nano-technology using biomedical applications and future prospects. International Journal of Pharmaceutical Sciences Review and Research, 31(2), 119-131.

Beshkar, F., Khojasteh, H., \& Salavati-Niasari, M. (2017). Recyclable magnetic superhydrophobic straw soot sponge for highly efficient oil/water separation. Journal of Colloid and Interface Science, 497, 57-65.

Burgos-Ramos, E., Martos-Moreno, G. A., Argente, J., \& Barrios, V. (2012). Multiplexed bead immunoassays: Advantages and limitations in pediatrics. In: Norman, H. L., Christopoulos, C., \& Christopoulos, T. K. (eds.). Advances in Immunoassay Technology.

Chen, C. H., Wen, C. P., \& Tsai, M. K. (2016). Fecal immunochemical test for colorectal cancer from a prospective cohort with 513,283 individuals: Providing detailed number needed to scope (NNS) before colonoscopy. Medicine (Baltimore), 95(36), e4414.

Choi, J. W., Oh, K. W., Thomas, J. H., Heineman, W. R., Halsall, H. B., Nevin, J. H., Helmicki, A. J., Henderson, H. T., \& Ahn, C. H. (2001). An integrated microfluidic biochemical detection system for protein analysis with magnetic bead-based sampling capabilities. Lab on a Chip, 2(1), 27-30.

Crespo, R. D., Elbaile, L., Carrizo, J., \& García, J. A. (2018). Optimizing the sensitivity of a GMR sensor for superparamagnetic nanoparticles detection: Micromagnetic simulation. Journal of Magnetism and Magnetic Materials, 446, 37-43.

Day, J. B., \& Basavanna, U. (2015). Magnetic bead based immuno-detection of Listeria monocytogenes and Listeria ivanovii from infant formula and leafy green vegetables using the Bio-Plex suspension array system. Food Microbiology, 46, 564-572.

De Roeck, Y., Philipse, E., Twickler, T. B., \& Van Gaal, L. (2017). Misdiagnosis of Graves' hyperthyroidism due to therapeutic biotin intervention. Acta Clinica Belgica, 1-5.

Dittmer, W. U., Evers, T. H., Hardeman, W. M., Huijnen, W., Kamps, R., de Kievit, P., Neijzen, J. H., Nieuwenhuis, J. H., Sijbers, M. J., Dekkers, D. W., Hefti, M. H., \& Martens, M. F. (2010). Rapid, high sensitivity, point-of-care test for cardiac troponin based on optomagnetic biosensor. Clinica Chimica Acta, 411(11-12), 868-873. 
Drung, D., Assmann, C., Beyer, J., Kirste, A., Peters, M., Ruede, F., \& Schurig, T. (2007). Highly sensitive and easy-to-use SQUID sensors. IEEE Transactions on Applied Superconductivity, 17(2), 699-704.

Eberbeck, D., Bergemann, C., Wiekhorst, F., Steinhoff, U., \& Trahms, L. (2008) Quantification of specific bindings of biomolecules by magnetorelaxometry. Journal of Nanobiotechnology, 6,4 .

Fert, A. (2008). Nobel lecture: Origin, development, and future of spintronics. Reviews of modem physics, 80, 1517-1530.

Foglia, S., Ledda, M., Fioretti, D., Iucci, G., Papi, M., Capellini, G., Grazia, L. M., Grimaldi, S., Rinaldi, M., \& Lisi, A. (2017). In vitro biocompatibility study of sub- $5 \mathrm{~nm}$ silica-coated magnetic iron oxide fluorescent nanoparticles for potential biomedical application. Scientific Reports, 7, 46513.

Galán, A., Comor, L., Horvatić, A., Kuleš, J., Guillemin, N., Mrljaka, V., \& Bhide, M. (2016). Library-based display technologies: Where do we stand? Molecular BioSystems, 12, 2342-2358.

Galkin, A. Y. (2014a). Modern methods of serological diagnosis of infectious and non-infectious diseases: Enzyme immunoassay, lateral flow immunoassay, immumoblot. Journal of Health Sciences, 4(16), 179-188.

Galkin, O. Y. (2014b). Porivnyal'na harakterystyka metodiv epitopnogo kartuvannya antygeniv proteyinovoyi pryrody [Comparative characteristic of methods of epitope mapping of antigens of protein nature]. Ukrainian Biochemical Journal, 86(4), 164-177 (in Ukrainian).

Galkin, A. Y., Komar, A. G., \& Grigorenko, A. A. (2015). Bioanalytical standardizing for serological diagnostic medical devices. Biotechnologia Acta, 8(2), 112-119.

Gehring, A. G., Irwin, P. L., Reed, S. A., Tua, S., Andreotti, P. E., \& AkhavanTafti, H. R. S. (2004). Enzyme-linked immunomagnetic chemiluminescent detection of Escherichia coli. Journal of Immunological Methods, 293, 97-106.

Ghodbane, S., Lahbib, A., Sakly, M., \& Abdelmelek, H. (2013). Bioeffects of static magnetic fields: Oxidative stress, genotoxic effects, and cancer studies. BioMed Research International, 2013, 602987.

Giannetto, M., Bianchi, M. V., Mattarozzi, M., \& Careri, M. (2017). Competitive amperometric immunosensor for determination of p53 protein in urine with carbon nanotubes/gold nanoparticles screen-printed electrodes: A potentia rapid and noninvasive screening tool for early diagnosis of urinary tract carcinoma. Analytica Chimica Acta, 991, 133-141.

Gorobets, Y. I., Gorobets, S. V., \& Gorobets, O. Y. (2013a). Biomineralization of intracellular biogenic magnetic nanoparticles and their expected functions. The Research Bulletin of the National Technical University of Ukraine “Kyiv Politechnic Institute”, 3, 28-33.

Gorobets, S. V., Gorobets, O. Y., \& Demyanenko, I. V. (2013b). Self-organization of magnetite nanoparticles in providing Saccharamyces cerevisiae yeasts with magnetic properties. Journal of Magnetism and Magnetic Materials, 337, 53-57.

Gorobets, O. Y., Gorobets, S. V., \& Gorobets, Y. I. (2014a). Biogenic magnetic nanoparticles: Biomineralization in prokaryotes and eukaryotes. In: Dekker encyclopedia of nanoscience and nanotechnology, Third Edition. Taylor and Francis, New York

Gorobets, O. Y., Gorobets, S. V., \& Sorokina, L. V. (2014b). Biomineralization and synthesis of biogenic magnetic nanoparticles and magnetosensitive inclusions in microorganisms and fungi. Functional Materials, 21(4), 427-436.

Gorobets, O., Gorobets, S., \& Koralewski, M. (2017). Physiological origin of biogenic magnetic nanoparticles in health and disease: From bacteria to humans. International Journal of Nanomedicine, 12, 4371-4395.

Han, C., Zhao, D., Deng, C., \& Hu, K. (2012). A facile hydrothermal synthesis of porous magnetite microspheres. Materials Letters, 70, 70-72.

Han, Y. A., Ju, J., Yoon, Y., \& Kim, S. M. (2014). Fabrication of cost-effective surface enhanced Raman spectroscopy substrate using glancing angle deposition for the detection of urea in body fluid. Journal of Nanoscience and Nanotechnology, 14(5), 3797-3799.

Hermanson, G. (2008). Bioconjugate techniques (3rd ed.). Academic Press.

Hoyoung, P., Hwang, M. P., \& Lee, K. H. (2013). Immunomagnetic nanoparticlebased assays for detection of biomarkers. Intemational Journal of Nanomedicine, 8, 4543-4552.

Issa, B., Obaidat, I. M., Albiss, B. A., \& Haik, Y. (2013). Magnetic nanoparticles: Surface effects and properties related to biomedicine applications. International Journal of Molecular Sciences, 14(11), 21266-21305.

Kala, M., Bajaj, K., \& Sinha, S. (1997). Magnetic bead enzyme-linked immunosorbent assay (ELISA) detects antigen-specific binding by phage-displayed scFv antibodies that are not detected with conventional ELISA. Analytical Biochemistry, 254, 263-266

Kim, D., Marchetti, F., Chen, Z., Zaric, S., Wilson, R. J., Hall, D. A., Gaster, R. S. Lee, J. R., Wang, J., Osterfeld, S. J., Yu, H., White, R. M., Blakely, W. F., Peterson, L. E., Bhatnagar, S., Mannion, B., Tseng, S., Roth, K., Coleman, M., Snijders, A. M., Wyrobek, A. J., \& Wang, S. X. (2013). Nanosensor dosimetry of mouse blood proteins after exposure to ionizing radiation. Scientific Reports, 3, 2234.
Kolhatkar, A. G., Dannongoda, C., Kourentzi, K., Jamison, A. C., Nekrashevich, I. Kar, A., Cacao, E., Strych, U., Rusakova, I., Martirosyan, K. S., Litvinov, D., Lee, T. R., \& Willson, R. C. (2015). Enzymatic synthesis of magnetic nanoparticles. International Journal of Molecular Sciences, 16(4), 7535-7550.

Konthur, Z., Wilde, J., \& Lim, T. S. (2010). Semi-automated magnetic beadbased antibody selection from phage display libraries. In: Kontermann, R., \& Dubel, S. (eds.). Antibody engineering. Vol. 1. Springer-Verlag Berlin Heidelberg, 267-287.

Kourilov, V., \& Steinitz, M. (2002). Magnetic-bead enzyme-linked immunosorbent assay verifies adsorption of ligand and epitope accessibility. Analytical Biochemistry, 311(2), 166-170.

Kurlyandskaya, G. V., Portnov, D. S., Beketov, I. V., Larrañaga, A., Safronov, A. P., Orue, I., Medvedev, A. I., Chlenova, A. A., Sanchez-Ilarduya, M. B., Martinez-Amesti, A., \& Svalov, A. V. (2017). Nanostructured materials for magnetic biosensing. Biochimica et Biophysica Acta, 1861(6), 1494-1506.

Li, D., Feng, S., Huang, H., Chen, W., Shi, H., Liu, N., Chen, L., Chen, W., Yu, Y., \& Chen, R. (2014). Label-free detection of blood plasma using silver nanoparticle based surface-enhanced Raman spectroscopy for esophageal cancer screening. Journal of Nanoscience and Nanotechnology, 10(3), 478-484.

Liao, S.-H., \& Su, Y.-K. (2017). Determining the time-dependent effective relaxation time of biofunctionalized magnetic nanoparticles conjugated with biotargets by using a high-Tc SQUID-based ac susceptometer for a magnetic immunoassay. Sensors and Actuators B: Chemical, 238, 66-70.

Lin, Y., Xu, G., Wei, F., Zhang, A., Yang, J., \& Hu, Q. (2016). Detection of CEA in human serum using surface-enhanced Raman spectroscopy coupled with antibody-modified $\mathrm{Au}$ and $\gamma-\mathrm{Fe}_{2} \mathrm{O}_{3} @ A u$ nanoparticles. Journal of Pharmaceutical and Biomedical Analysis, 121, 135-140.

Lin, Y. H., Chen, Y. J., Lai, C. S., Chen, Y. T., Chen, C. L., Yu, J. S., \& Chang Y. S. (2013). A negative-pressure-driven microfluidic chip for the rapid detection of a bladder cancer biomarker in urine using bead-based enzymelinked immunosorbent assay. Biomicrofluidics, 7(2), 24103.

Luo, S., Liu, Y., Rao, H., Wang, Y., \& Wang, X. (2017). Fluorescence and mag netic nanocomposite $\mathrm{Fe}_{3} \mathrm{O}_{4} @ \mathrm{SiO}_{2} @ \mathrm{Au} \mathrm{MNPs}$ as peroxidase mimetics for glucose detection. Analytical Biochemistry, 538, 26-33.

Lutsenko, T. N., Kovalenko, M. V., \& Galkin, O. Y. (2017). Validation of biological activity testing procedure of recombinant human interleukin-7. Ukrainian Biochemical Journal, 89(1), 82-89.

Malou, N., \& Raoult, D. (2011). Immuno-PCR: A promising ultrasensitive diagnostic method to detect antigens and antibodies. Trends in Microbiology, 19(6), 295-302.

Manera, M. G., Pellegrini, G., Lupo, P., Bello, V., Fernández, C. J., Casoli, F., Rella, S., Malitesta, C., Albertini, F., Mattei, G., \& Rella, R. (2017). Functional magneto-plasmonic biosensors transducers: Modelling and nanoscale analysis. Sensors and Actuators B: Chemical, 239, 100-112.

Mani, V., Chikkaveeraiah, B. V., \& Rusling, J. F. (2011). Magnetic particles in ultrasensitive biomarker protein measurements for cancer detection and monitoring. Expert Opinion on Medical Diagnostics, 5(5), 381-391.

McConnell, S. J., Dinh, T., Le, M. H., \& Spinella, D. G. (1999). Biopanning phage display libraries using magnetic beads vs. polystyrene plates. Biotechniques, 26(208-10), 214

Mohapatra, M., \& Anand, S. (2010). Synthesis and applications of nanostructured iron oxides/hydroxides. International Journal of Engineering, Science and Technology, 2(8), 127-146.

Morozov, V. N., Groves, S., Turell, M. J., \& Bailey, C. (2007). Three minuteslong electrophoretically assisted zeptomolar microfluidic immunoassay with magnetic-beads detection. Journal of the American Chemical Society, 129(42), 12628-12629.

Mulvaney, S. P., Cole, C. L., Kniller, M. D., Malito, M., Tamanaha, C. R., Rife, J. C., Stanton, M. W., \& Whitman, L. (2007). Rapid, femtomolar bioassays in complex matrices combining microfluidics and magnetoelectronics. Journal of Biosensors and Bioelectronics, 23(2), 191-200.

Mulvaney, S. P., Myers, K. M., Sheehan, P. E., \& Whitman, L. J. (2009). Attomolar protein detection in complex sample matrices with semi-homogeneous fluidic force discrimination assays. Journal of Biosensors and Bioelectronics, 24(5), 1109-1115.

Nagasaki, Y., Kobayashi, H., Katsuyama, Y., Jomura, T., \& Sakura, T. (2007) Enhanced immunoresponse of antibody/mixed-PEG co-immobilized surface construction of high-performance immunomagnetic ELISA system. Journal of Colloid and Interface Science, 309(2), 524-530.

Nakatani, Y., Hayashi, T., Miyato, Y., \& Itozaki, H. (2012). Laser SQUID microscope for the evaluation of solar cell. Physics Procedia, 36, 394-399.

Nie, Y., Zhang, P., Wang, H., Zhuo, Y., Chai, Y. Q., \& Yuan, R. (2017). An ultrasensitive electrochemiluminescence biosensing platform for detection of multiple types of biomarkers toward identical cancer on a single interface. Analytical Chemistry.

Nikitin, P., Ksenevich, T., Nikitin, M., \& Gorshkov, B. (2008a). Opto-magnetic assays based on magnetic nanoparticles and optical label-free biosensors. 
Proceedings of Ninth European Conference on Optical Chemical Sensors and Biosensors, EUROPT(R)ODE IX, Dublin, P. OA4.2.

Nikitin, M. P., Tomo, M., Chen, H., Rosengart, A., \& Nikitin P. I. (2008b). Quantitative real-time in vivo detection of magnetic nanoparticles by their nonlinear magnetization. Journal of Applied Physics, 103(7), 07A304.

Nikitin, M. P., Orlov, A. V., Znoyko, S. L., Bragina, V. A., Gorshkov, B. G., Ksenevich, T. I., Cherkasov, V. R., \& Nikitin, P. I. (2017). Multiplex biosensing with highly sensitive magnetic nanoparticle quantification method. Journal of Magnetism and Magnetic Materials, In press.

Orlov, A. V., Khodakova, J. A., Nikitin, M. P., Shepelyakovskaya, A. O., Brovko, F. A., Laman, A. G., Grishin, E. V., \& Nikitin, P. I. (2013). Magnetic immunoassay for detection of staphylococcal toxins in complex media. Analytical Chemistry, 85(2), 1154-1163.

Orlov, A. V. (2014). Razrabotka metodov immunoanaliza s ispol'zovaniem magnitnyih nanomarkerov [Development of immunoanalization methods using magnetic nanomarkers]. Moscow (in Russian).

Pallaoro, A., Hoonejani, M. R., Braun, G. B., Meinhart, C. D., \& Moskovits, M. (2015). Rapid identification by surface-enhanced Raman spectroscopy of cancer cells at low concentrations flowing in a microfluidic channel. ACS Nano, 9(4), 4328-4336.

Park, J. (2016). A giant magnetoresistive reader platform for quantitative lateral flow immunoassays. Sensors and Actuators A: Physical, 250, 55-59.

Philippova, O., Barabanova, A., Molchanov, V., \& Khokhlov, A. (2011). Magnetic polymer beads: Recent trends and developments in synthetic design and applications. European Polymer Journal, 47(4), 542-559.

Rajput, S., Pittman, C. U., \& Mohan, D. (2016). Magnetic magnetite $\left(\mathrm{Fe}_{3} \mathrm{O}_{4}\right)$ nanoparticle synthesis and applications for lead $\left(\mathrm{Pb}^{2+}\right)$ and chromium $\left(\mathrm{Cr}^{6+}\right)$ removal from water. Journal of Colloid and Interface Science, 468, 334-346.

Rizzi, G., Lee, J. R., Guldberg, P., Dufva, M., Wang, S. X., \& Hansen, M. F. (2017). Denaturation strategies for detection of double stranded PCR products on GMR magnetic biosensor array. Journal of Biosensors and Bioelectronics, 93, 155-160.

Rong, Z., Wang, C., Wang, J., Wang, D., Xiao, R., \& Wang, S. (2016). Magnetic immunoassay for cancer biomarker detection based on surface-enhanced resonance Raman scattering from coupled plasmonic nanostructures. Biosensors and Bioelectronics, 84, 15-21.

Rusling, J. F., Kumar, C. V., Gutkind, J. S., \& Patele, V. (2010). Measurement of biomarker proteins for point-of-care early detection and monitoring of cancer. Analyst, 135(10), 2496-2511.

Saari, M. M., Tsukamoto, Y., Kusaka, T., Ishihara, Y., Sakai, K., Kiwa, T., \& Tsukada, K. (2015). Effect of diamagnetic contribution of water on harmonics distribution in a dilute solution of iron oxide nanoparticles measured using high-Tc SQUID magnetometer. Journal of Magnetism and Magnetic Materials, 394, 260-265.

Salek-Maghsoudi, A., Vakhshiteh, F., Torabi, R., Hassani, S., Ganjali, M. R., Norouzi, P., Hosseini, M., \& Abdollahi, M. (2018). Recent advances in biosensor technology in assessment of early diabetes biomarkers. Journal of Biosensors and Bioelectronics, 99, 122-135.

Santhoshkumar, J., Rajeshkumar, S., \& Venkat, K. S. (2017). Phyto-assisted synthesis, characterization and applications of gold nanoparticles. Biochemistry and Biophysics Reports, 11, 46-57.

Shevchenko, K. G., Cherkasov, V. R., Tregubov, A. A., Nikitin, P. I., \& Nikitin, M. P. (2017). Surface plasmon resonance as a tool for investigation of noncovalent nanoparticle interactions in heterogeneous self-assembly and disassembly systems. Journal of Biosensors and Bioelectronics, 88, 3-8.

Shipunova, V. O., Nikitin, M. P., Lizunova, A. A., Ermakova, M. A., Deyev, S. M., \& Petrov, R. V. (2013). Polyethyleneimine-coated magnetic nanoparticles for cell labeling and modification. Doklady Biochemistry and Biophysics, 452(1), 245-247.

Shlyapnikov, Y. M., Shlyapnikova, E. A., Simonova, M. A., Shepelyakovskaya, A. O., Brovko, F. A., Komaleva, R. L., Grishin, E. V., \& Morozov, V. N. (2012). Rapid simultaneous ultrasensitive immunodetection of five bacterial toxins. Analytical Chemistry, 84(13), 5596-5603.

Smith-Bindman, R., Miglioretti, D. L., Johnson, E., Lee, C., Feigelson, H. S., Flynn, M., Greenlee, R. T., Kruger, R. L., Hombrook, M. C., Roblin, D., Solberg, L. I., Vanneman, N., Weinmann, S., \& Williams, A. E. (2012). Use of diagnostic imaging studies and associated radiation exposure for patients enrolled in large integrated health care systems, 1996-2010. Journal of the American Medical Association, 307(22), 2400-2409.

Sood, A., Arora, V., Shah, J., Kotnala, R. K., \& Jain, T. K. (2017). Multifunctional gold coated iron oxide core-shell nanoparticles stabilized using thiolated sodium alginate for biomedical applications. Materials Science and Engineering. C, Materials for Biological Applications, 80, 274-281.
Svobodova, Z., Krulisova, P., Cerna, M., Jankovicova, B., \& Bilkova, Z. (2015). On-chip ELISA on magnetic particles: Isolation and detection of specific antibodies from serum. Nanocon 2015: 7th International Conference, Brno, Czech Republic, EU.

Tang, D., Yuan, R., \& Chai, Y. (2007). Magnetic control of an electrochemical microfluidic device with an arrayed immunosensor for simultaneous multiple immunoassays. Clinical Chemistry, 53(7), 1323-1329.

Tang, H., \& Han, D. (2017). Controllable preparation of iron nanostructures and their magnetic properties. Journal of Magnetism and Magnetic Materials, 444, 125-131.

Tsai, H. Y., Hsu, C. F., Chiu, I. W., \& Fuh, C. B. (2007). Detection of C-reactive protein based on immunoassay using antibody-conjugated magnetic nanoparticles. Analytical Chemistry, 79(21), 8416-8419.

Tygai, Y. I., \& Besarab, A. B. (2014). The mathematical model of voltage transformers for the study of ferroresonant processes. IEEE International Conference on Intelligent Energy and Power Systems, Conference Proceedings, 77-80.

Vesanen, P. T., Nieminen, J. O., Zevenhoven, K. C., Dabek, J., Parkkonen, L. T. Zhdanov, A. V., Luomahaara, J., Hassel, J., Penttilä, J., Simola, J., Ahonen, A. I., Mäkelä, J. P., \& Ilmoniemi, R. J. (2013). Hybrid ultra-low-field MRI and magnetoencephalography system based on a commercial whole-head neuromagnetometer. Magnetic Resonance in Medicine, 69(6), 1795-1804.

Vidojkovic, S. M., \& Rakin, M. P. (2017). Surface properties of magnetite in high temperature aqueous electrolyte solutions: A review. Advances in Colloid and Interface Science, 245, 108-129.

Wacker, R., Ceyhan, B., Alhorn, P., Schueler, D., Lang, C., \& Niemeyer, C. M. (2007). Magneto immuno-PCR: A novel immunoassay based on biogenic magnetosome nanoparticles. Biochemical and Biophysical Research Communications, 357(2), 391-396.

Wang, S., Zhang, Y., An, W., Wei, Y., Liu, N., Chen, Y., \& Shuang, S. (2015). Magnetic relaxation switch immunosensor for the rapid detection of the foodborne pathogen Salmonella enterica in milk samples. Food Control, 55, 43-48.

Wang, W., Ma, P., Dong, H., Krause, H. J., Zhang, Y., Willbold, D., Offenhaeusser, A., \& Gu, Z. (2016). A magnetic nanoparticles relaxation sensor for protein-protein interaction detection at ultra-low magnetic field. Biosensors and Bioelectronics, 80, 661-665.

Wang, T., Zhou, Y., Lei, C., Luo, J., Xie, S., \& Pu, H. (2017a). Magnetic impedance biosensor: A review. Biosensors and Bioelectronics, 90, 418-435.

Wang, Y., Zhao, G., Li, X., Liu, L., Cao, W., \& Wei, Q. (2017b). Electrochemiluminescent competitive immunosensor based on polyethyleneimine capped $\mathrm{SiO}_{2}$ nanomaterials as labels to release $\mathrm{Ru}(\mathrm{bpy})_{3}{ }^{2+}$ fixed in 3D Cu/Ni oxalate for the detection of aflatoxin $B_{1}$. Journal of Biosensors and Bioelectronics, 101, 290-296.

Wu, W., Wu, Z., Yu, T., Jiang, C., \& Kim, W. S. (2015). Recent progress on magnetic iron oxide nanoparticles: Synthesis, surface functional strategies and biomedical applications. Science and Technology of Advanced Materials, 16(2), 023501.

Wu, J., Pei, L., Xuan, S., Yan, Q., \& Gong, X. (2016). Particle size dependent rheological property in magnetic fluid. Joumal of Magnetism and Magnetic Materials, 408, 18-25.

Xu, X., Li, H., Hasan, D., Ruoff, R. S., Wang, A. X., \& Fan, D. L. (2013). Nearfield enhanced plasmonic-magnetic bifunctional nanotubes for single cell bioanalysis. Advanced Functional Materials, 23(35), 4332-4338.

Xu, Z., Jiang, J., Wang, X., Han, K., Ameen, A., Khan, I., Chang, T. W., \& Liu, L. (2016). Large-area, uniform and low-cost dual-mode plasmonic naked-eye colorimetry and SERS sensor with handheld Raman spectrometer. Nanoscale, 8, 6162-6172.

Xue, P., Sun, L., Li, Q., Zhang, L., Guo, J., Xu, Z., \& Kang, Y. (2017). PEGylated polydopamine-coated magnetic nanoparticles for combined targeted chemotherapy and photothermal ablation of tumour cells. Colloids and Surfaces B: Biointerfaces, 160, 11-21.

Yang, J., Palla, M., Bosco, F. G., Rindzevicius, T., Alstrøm, T. S., Schmidt, M. S., Boisen, A., Ju, J., \& Lin, Q. (2013). Surface-enhanced Raman spectroscopy based quantitative bioassay on aptamer-functionalized nanopillars using large-area Raman mapping. ACS Nano, 7(6), 5350-5359.

Zhang, S., Lu, T., Qi, D., Cao, Z., Zhang, Z., \& Zhao, H. (2017). Synthesis of quaternized chitosan-coated magnetic nanoparticles for oil-water separation. Materials Letters, 191, 128-131.

Zhu, Y., Kekalo, K., NDong, C., Huang, Y., Shubitidze, F., Griswold, K., Baker, I., \& Zhang, J. (2016). Magnetic-nanoparticle-based immunoassays-on-chip: Materials synthesis, surface functionalization, and cancer cell screening. Advanced Functional Materials, 26(22), 3953-3972. 